\title{
Écoutons les chants de Takuu
}

\section{Gilles Bounoure}

\section{(2) OpenEdition \\ Journals}

Édition électronique

URL : http://journals.openedition.org/jso/6174

DOI : 10.4000/jso.6174

ISSN : 1760-7256

\section{Éditeur}

Société des océanistes

\section{Édition imprimée}

Date de publication : 15 décembre 2010

Pagination : 229-234

ISBN : 978-2-85430-027-7

ISSN : 0300-953x

\section{Référence électronique}

Gilles Bounoure, «Écoutons les chants de Takuu », Journal de la Société des Océanistes [En ligne], 130-131 | 2010, mis en ligne le 15 décembre 2010, consulté le 24 septembre 2020. URL : http:// journals.openedition.org/jso/6174; DOI : https://doi.org/10.4000/jso.6174

(C) Tous droits réservés 


\title{
Écoutons les chants de Takuu
}

par

\author{
Gilles BOUNOURE*
}

À propos de :

Richard MoyLe, 2007. Songs from the Second Float. A Musical Ethnography of Takú Atoll, Papua New Guinea, Honolulu, University of Hawai'i Press, Pacific Islands Monograph Series 21, XXIV-310 p., glossaire, bibliogr., index, cartes, schémas, nombreuses ill. dans le texte.

Richard Moyle, 2005. Songs from the Second Float. Music from the Island of Takú. World Premiere recording, CD Manu 2042, Auckland, ODE Record Company.

Peu de citoyens de Papouasie NouvelleGuinée connaissent l'existence de l'atoll de Takú (jadis dénommé Marqueen ou Mortlock, nom que porte aussi un groupe d'îles de Micronésie), enclave polynésienne isolée au nord-est des trois millions de $\mathrm{km}^{2}$ de zone maritime que compte leur État. Cinq à six cents habitants y vivent aujourd'hui, mais sans certitude de pouvoir s'y maintenir. Leur histoire récente est ponctuée de drames. Avant le passage des Blancs, ils devaient être quelque deux à trois cents, si l'on se fie au nombre et à la taille des grands canots de pêche aux proues ouvragées grâce auxquels ils allaient pêcher au-delà du lagon les poissons les plus prestigieux, thons, requins et Ruvettus pretiosus ${ }^{1}$. Ils n'étaient plus que cinquante en 1885 (Parkinson, 1887-1888:209), et une décennie plus tard, dix-sept sur une photographie les réunissant tous (et peut-être due aussi à Parkinson, qui parle d'environ « 20 Seelen » en 1896, dans son livre de 1907, p. 517 ; photo reproduite par R. Moyle p. 19). En 1913, ils n'étaient plus que neuf, selon un témoignage peu connu sur lequel on reviendra (Jacques, 1922 : 160). Dès 1884, les grands canots ne servaient plus (Churchill, 1909: 88).

Avant les baleiniers (dont les insulaires de Takú auraient réussi à tuer un équipage et à faire disparaître le navire selon Parkinson [ibid. : 534], voir R. Moyle, 2007 : 30-32 et 276 note 21), il y eut les pêcheurs d'holothuries à destination du marché chinois, pour qui la qualité insurpassable des "bêches-de-mer" de ces eaux-là justifiait toutes les ruses et toutes les violences. Autre « enclave polynésienne » de cette même province des Salomon du Nord, les îles Carteret (Kilinailau) portèrent longtemps dans la littérature occidentale le nom d'îles « du Massacre » après le passage du capitaine Benjamin Morrell en 1830 et ses coups de force pour établir sur un des points de cet atoll son hangar de préparation et de séchage du " trépang »-épisode que Dumont d'Urville lui-même relate avec un certain dégoût (1835: 163-168). Pour Takú, le récit apologétique qu'a laissé Andrew Cheyne de sa campagne de pêche (Shineberg, 1971 :295-302) de la fin décembre 1843 à la fin janvier 1844 en dit long non pas sur les insulaires, mais sur les précautions prises contre eux, sauf à les massacrer, pour pouvoir piller le lagon et couper des arbres sans leur accord : palissades, tranchées, sentinelles... Comment ces visites qui n'étaient pas de courtoisie diffusèrent les maladies occidentales à Takú, on ne peut que le supposer, faute de sources écrites là comme ailleurs, mais personne ne saurait attribuer aujourd'hui avec Churchill

1. Sur ces canots et cette dernière espèce de poisson d'eau profonde généralement pêchée la nuit, voir Parkinson (1907 : 536-539) et Churchill (1909 : 88) ; sur les chants associés à cette pêche, voir Moyle (2007: 63, 130, 187, 245).

* flongue@wanadoo.fr 
(1909: 92) cette dépopulation dramatique à la « nature sans pitié » et à une « lutte pour la vie» trop inégale pour les insulaires.

Les avanies ne s'arrêtèrent pas là. Il semble peu douteux que Parkinson, même s'il ne s'en est pas vanté, aura parlé à sa belle-sœur la « reine Emma » de la dépopulation de l'atoll qu'il venait de constater et qu'il lui aura suggéré d'installer un « comptoir » dans son île principale, quitte à en déplacer les quelques habitants sur un îlot voisin, Kapeiatu. Moyennant quatre haches et dix livres de tabac, elle procédait à «l'achat» de l'ensemble de l'atoll dès 1886 , mais la mise en exploitation réclama quelques années de plus, nouvelles plantations, déportation des insulaires, installation de travailleurs venus de Buka, de Nouvelle-Bretagne et même de l'Amirauté. La crise du coprah et la prise de contrôle en 1914 par les autorités australiennes de cet atoll sous administration allemande depuis trente ans ne compromirent pas le fonctionnement de la plantation principale, passée par expropriation aux mains d'un Écossais en 1926. Deux visites faites à l'atoll par Chinnery, récemment nommé anthropologue gouvernemental pour la Nouvelle-Guinée sous mandat, lui révélèrent le sort inique des insulaires, devenus plus nombreux du fait de la présence des travailleurs immigrés. En 1930, il obtenait de la Cour centrale de Rabaul un jugement restituant l'atoll aux insulaires, tandis qu'avec leur accord le planteur écossais commençait à leur bâtir des habitations individuelles (au lieu de leurs «maisons longues ») sur un autre îlot, Nukutoa (environ $4 \mathrm{ha}$ ), plus vaste que leur précédent refuge Kapeiatu, qu'ils avaient surnommé le «flotteur » de leur ancien "canot ", l'île principale Takú (60 ha). Nukutoa est ainsi le « second flotteur » sur lequel ils vivent depuis lors et où $\mathrm{R}$. Moyle est allé recueillir et étudier leurs chants et leurs danses, désormais connus par son $\mathrm{CD}$ et par son livre.

Or voilà que depuis le début de ce millénaire au moins l'atoll est menacé d'engloutissement par l'élévation du niveau de l'océan, voire par la subsidence du cône volcanique qui le porte, point sur lequel les géophysiciens débattent encore. R. Moyle n'est pas le dernier à avoir donné l'alerte (voir par exemple Wane, 2005) et il semble même avoir inspiré directement l'entreprise de Briar March, jeune cinéaste néozélandaise qui, séjournant à Takú entre 2007 et 2009, en a rapporté un documentaire saisissant,
Te Henua e Noho. There once was an island, récemment projeté avec succès dans plusieurs grandes villes du Pacifique. On y voit les effets de la tempête de l'hiver 2008, les jardins envahis par l'eau de mer et le dépérissement des taros géants, les efforts constants des insulaires pour édifier des digues de fortune, le désarroi parfois violent des jeunes gens sans perspective d'avenir, "si perdus qu'il n'y a pas à les blâmer», et le désespoir de leurs aînés décidés à mourir sur leur île même si le gouvernement leur offrait de les reloger à Bougainville, comme il est prévu pour les habitants de Carteret, eux aussi menacés de submersion.

Tel est le dernier drame dont pourrait ne pas se relever la petite société de Takú, après deux siècles d'une résistance exceptionnelle que R. Moyle souligne à merveille dans l'introduction et le premier chapitre remarquablement documenté de son livre (Geography and History, pp. 9-46). Il est admirable que les insulaires aient réussi à sauvegarder et à enrichir leur patrimoine musical, poétique et festif, alors qu'ils s'étaient fait écarter des lieux sacrés de leur île principale et dérober trois effigies d'esprits ancestraux, aujourd'hui conservées au musée de Leipzig (Moyle, 2000 ; voir aussi Treide, 1997: 57 et $\mathrm{n}^{\mathrm{o}} 177$ à 179). Contrairement à ce qu'écrit R. Moyle (2007 : 276 n. 23, d'après Buschmann, 2000), il n'y a pas de doute que ces sculptures furent emportées par Karl Nauer, capitaine du vapeur Sumatra de la Norddeutscher Lloyd, lors de l'une de ses tournées régulières pour charger du coprah (voir notamment Burger, 1923 : chap. x). Lors de ce qui fut probablement la dernière, en 1913, il avait à son bord Norbert Jacques (1922 : 155-160 et planche faisant face à la p. 145), voyageur, écrivain et photographe luxembourgeois qui a consigné quelques observations désolées (Sterbende Völker, " peuples à l'agonie », écrit-il) et pris deux photographies qu'on a jugé utile de reproduire ci-dessous puisque ce témoignage semble ignoré des spécialistes de Takú et que ces deux documents pourraient également ne pas être connus des insulaires actuels.

À côté de ces trois sculptures dont les insulaires de Takú pourraient exiger la restitution comme l'envisageait R. Moyle (2000 : 107), un autre objet sacré qu'il ne mentionne pas fut « acquis » par Parkinson (1907: 527 et Abb. 86, même page), une «lance sculptée » de motifs 

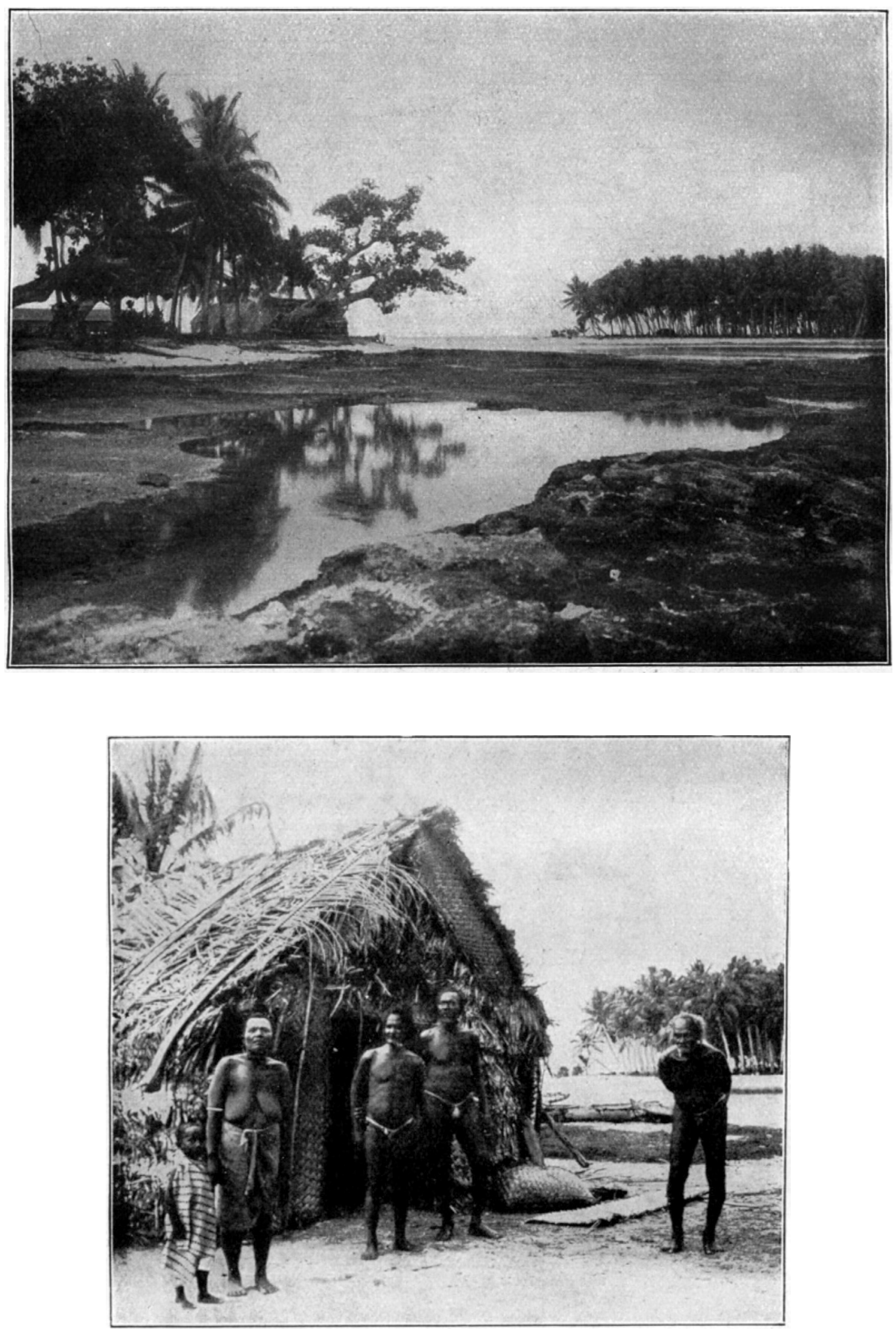

FIGURE 1. - « Dernières huttes et derniers indigènes des îles Mortlock », vues prises par Norbert Jacques en 1913 à Takú (D.R.) 


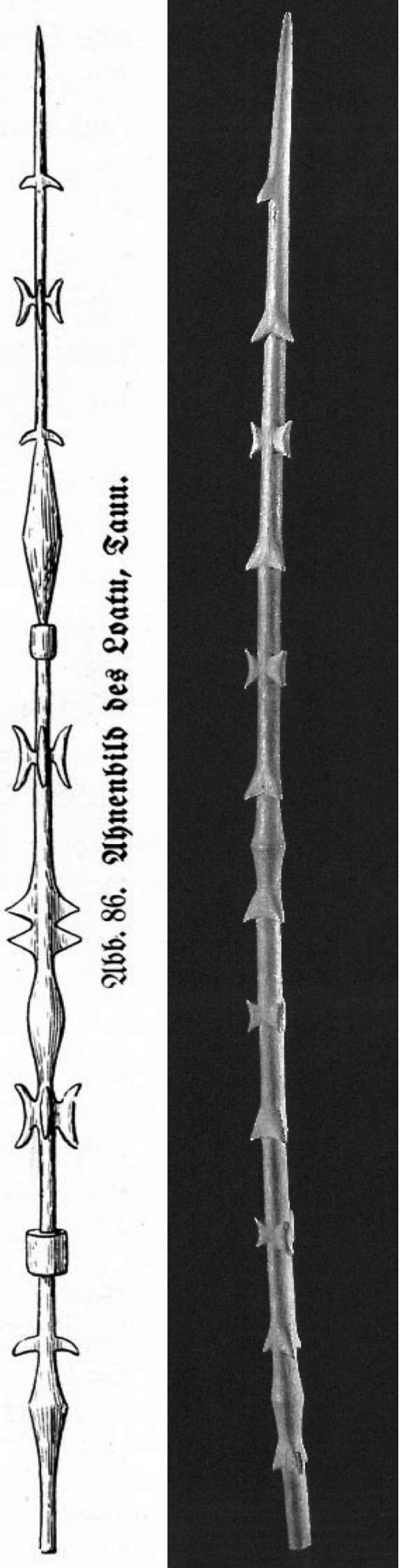

FIgURE 2 a et b. - a) « Loatu », d'après Parkinson (1907: 527) (D. R.) ; b) « Loatu staff god», vente Sotheby's (Photo (C) Sotheby's)
« géométriques » en ronde-bosse, représentant d'après lui «l'ancêtre Loatu » vénéré dans la «maison des esprits» (hare aiku) de Takú. Aucune trace ne semble subsister dans les collections publiques de cette " vieille pièce » qui rappelle les staff gods, godsticks ou « bâtons généalogiques " de Polynésie, de Santa Cruz et d'autres cultures apparentées. En 2001, un spécimen assez comparable apparaissait sur le marché de l'art (vente Sotheby's New York du 19 mai 2001, lance sculptée, " A rare Loatu staff god», lot 66 adjugé 12000 dollars, et, précise Philippe Peltier, « acheté par le musée du quai Branly sur les conseils de Douglas Newton ", $\mathrm{n}^{\circ}$ d'inventaire 70.2001.21.1) ; la publication du catalogue des collections polynésiennes et micronésiennes du Rautenstrauch-Joest Museum de Cologne révélait quelques autres menus objets acquis auprès de Phoebe Altmann, la nièce de « la reine Emma » devenue propriétaire de la plantation de Takú (Thode-Arora, 2001 : 372-375, 14-15). De telles publications laissent espérer, avec les efforts de R. Moyle, d'autres spécialistes, de passionnés comme Briar March et son équipe, et de groupes animés par des expatriés (notamment la Takú Islands association), un début de recension des vestiges matériels de cette civilisation méconnue mais exemplaire par sa vitalité, jusqu'à ce que l'actuelle menace de submersion y porte le désarroi.

Aucun chant ne témoigne des quatre décennies de résidence et de travail forcés sur le « premier flotteur ", Kapeiatu, au cours desquelles se disloqua l'ancienne organisation de la société en deux côtés ou groupes (hata) et cinq clans ou « maisons » (hare). Le tempérament plus libéral du planteur écossais, les mesures de protection prises par Chinnery et l'isolement de l'atoll, à $250 \mathrm{~km}$ au nord-est de Kieta et 15 à 18 heures de navigation, permirent aux insulaires de se doter d'un rythme propre et de retrouver leur goût du chant, sans interférence des marchands ni surtout des missionnaires, à la différence de Nukumanu, de Nuguria (Moyle, 2007 : 4), de Tikopia (Firth, 1990 : 11-12), des Ellice ou Tuvalu (Koch, $2000: 19-20)$ et de maintes autres communautés venues à substituer le gospel à leurs vieux chants. Les deux églises de Nukutoa ne furent bâties qu'en 1999 et si les huit séjours de R. Moyle à Takú entre 1994 et la parution de son livre se firent à la demande des insulaires, il lui fallut aussi l'accord des autorités sanitaires et politiques alors occupées par la rébellion partie de la mine de Panguna, à quelques kilomètres de Kieta. Comme il l'a relaté à une journaliste (Wane, 2005), l'ethnomusicologue dut s'impro- 
viser chirurgien sur cet atoll sans autre ressource médicale que la visite annuelle d'un dentiste, et recoudre au fil dentaire la main d'un pêcheur victime d'un requin.

« À Takú, le chant n'est pas une activité relevant du spectacle ou du théâtre pour le plaisir de l'auditoire, mais une expression introspective des valeurs et des croyances permettant aux habitants de fonctionner en tant que communauté sur le plan domestique aussi bien que rituel. »

Ainsi s'ouvre le passionnant chapitre 2 : Takú Society as the Locus for Musicking (pp. 47-110) où R. Moyle démontre, en dépit de toutes les transformations subies en un siècle et demi, le rôle collectif essentiel constamment dévolu au chant dans cette société combinant « coopérations à long terme et compétitions de courte durée », comme les régates dans le lagon (il y en aurait eu une vingtaine en 1998) ou les concours de pêche dont les prises sont partagées par l'ensemble de la collectivité. Un tel « égalitarisme » (p. $83 s q$.), que font également régner les femmes lorsqu'elles se déguisent en policiers et en magistrats à perruque pour contrôler les compétiteurs masculins ou quand elles se chargent de distribuer équitablement prises et récoltes, résulte-t-il de l'histoire récente des habitants de Takú ? Faut-il en croire Parkinson (1907 : 528) les faisant se répartir jadis en trois " classes ", comme partout ailleurs dans les «îles orientales » qu'il décrit ? L'analyse que fait R. Moyle du rôle de l'Ariki, des chefs de clan et des autres «leaders» (pp. 50-64) vient confirmer au contraire que l'égalitarisme est « une caractéristique importante des communautés des atolls polynésiens ", peut-être de nature à expliquer l'importance qu'on y accordait au chant collectif, pour faire face à des conditions de vie particulièrement difficiles (Koch, $2000: 21$ ).

Les quatre chapitres suivants, spécifiquement consacrés à la musique et à la danse, offrent également des développements de premier intérêt sur les transformations et les permanences observables dans le domaine religieux (3: Religious Contexts of Music) et dans celui de la composition musicale et poétique (4 : Processes of Taku Music). Elle peut être d'origine surnaturelle ou humaine, et dans ce dernier cas elle est sujette à des renouvellements plus fréquents, mais aussi à des critiques nettement argumentées. Les pages (150-156) où R. Moyle a recueilli de telles remarques sur les tuki (terme désignant les chants mais aussi le tambour les accompagnant), apparemment sans équivalent dans les recherches publiées, suggèrent l'importance et le raffinement des critères esthétiques en vigueur à
Takú. En décrivant les divers types de chants et de danses ( 5 : The Nature of Takú Song ; 6 : The Nature of Takú Dance), R. Moyle ne fait que très exceptionnellement appel à des parallèles polynésiens ou micronésiens, sauf lorsqu'il s'agit d'emprunts évidents. Il évite de même d'entrer dans l'épineux débat des origines de la population de Takú, ou de sa langue dont il avait cru d'abord qu'elle s'apparentait au samoan, pour découvrir " cinq minutes après avoir débarqué » sur l'atoll qu'elle s'en écartait grandement (Wane, 2005).

L'écoute du CD préalablement édité par R. Moyle doit compléter la lecture de son beau livre, ces enregistrements faisant évidemment ressentir de façon plus directe «l'expression introspective » des chanteurs et des danseurs dont parle son ouvrage. Mais à la différence des disques, cassettes ou CD accompagnant des publications comparables (par exemple Christensen, 1964 ; Firth, 1990 ; Koch, 2000) sans autre ajout documentaire, celui-ci propose des chants non recueillis dans le volume imprimé. Tel le cas du rue de la plage 1 , du tuki venu d'Ontong Java de la plage 2, ou encore du fragment de $l u$ monodique de la plage 6 , forme de chant "préhistorique » qui ne se trouve qu'à Takú selon R. Moyle. La variété des chants et des danses encore en usage est bien suggérée par ces douze plages, depuis le tuki évoquant vraisemblablement le séjour de Cheyne sur l'atoll en 1843 ( $\mathrm{n}^{\circ} 4$, voir p. 34 du livre) et la danse féminine manakoho inspirée par un esprit $\left(\mathrm{n}^{\circ} 3\right.$, p. 230 du livre), jusqu'au tuki final, créé en 1997 en hommage à un pêcheur décédé depuis peu $\left(\mathrm{n}^{\circ}\right.$ 12). C'est la structure particulière des chants et des danses de Takú, limitant les efforts de mémorisation, qui a permis le développement d'un si vaste répertoire (p. 271). «Il y a plus d'une centaine de rue (chants et danses d'hommes) dans le répertoire actuel », écrit R. Moyle dans le livret édité avec ce $\mathrm{CD}$. S'il a été contraint, là comme dans son livre, de se borner à un petit nombre d'exemples pour chaque type de chant et de danse, ils sont suffisamment parlants et émouvants pour faire souhaiter l'édition de tout ce qu'il a pu recueillir de ce patrimoine encore vivant mais menacé de toutes parts, et dont l'oubli ajouterait un drame de plus à l'histoire de Takú.

En quoi peuvent-ils émouvoir l'Européen qui les écoutera ? Poignante et exemplaire, la situation actuelle des insulaires de Takú luttant contre l'avancée de la mer se retrouve cependant sur maintes autres « îles basses » du Pacifique en passe de devenir elles aussi invivables, et nombre d'entre elles ont également connu des déplacements forcés de population à l'époque coloniale 
(voir par exemple Maude, 1968). Dans le dernier paragraphe de son livre consacré aux chants de Tikopia (1990 : 296-297), R. Firth évoquait la "puissante expérience esthétique » que lui avaient procurée l'écoute et la récitation de leurs paroles, "stimulant [son] imagination jusqu'à l'émotion " peut-être au risque, ajoutait-il, de céder au "piège de l'exotisme ». Indépendamment des observateurs occidentaux et de leurs « expériences esthétiques », il se pourrait cependant que la capacité d'émotion des chants de la Polynésie occidentale et des enclaves polynésiennes soit due en partie au caractère généralement retenu de leur expression verbale, même à propos des événements les plus dramatiques, dont la mention explicite est fréquemment évitée (Koch, 2000 : 17), comme si les poèmes servaient de condensateurs à l'énergie des chants et des danses. R. Firth a lui-même relevé cette propension à l'allusion, à l'understatement, aux expressions à double entente saisissables seulement par les membres de la communauté, voire par une petite partie d'entre eux. On en trouvera de nombreuses confirmations à Takú, par exemple avec l'existence de certaines chansons destinées à un auditoire limité ou réservées aux chanteurs d'un clan déterminé (Moyle, 2007 : 192). De ce point de vue, chants et danses ont apparemment constitué pour ces sociétés un moyen de préservation et de résistance, et les insulaires de Takú s'en seront admirablement servis avant la submersion annoncée de leur atoll.

\section{BIBLIOGRAPHIE}

BURGER Friedrich, 1923. Unter den Kannibalen der Südsee, Studienreise durch die Melanesische Inseln, Dresden, Verlag Deutsche Buchwerkstätten.

Buschmann Rainer, 2000. Karl Nauer and the Politics of Collecting Ethnographic Objects in German New Guinea, Pacific Arts 21-22, pp. 93-102.
Christensen Dieter und Gerd Koch, 1964. Die Musik der Ellice-Inseln, Berlin, Museum für Völkerkunde.

Churchill William, 1909. The Dying People of Takú, Bulletin of the American Geographical Society 41-2, pp. 86-92.

Dumont D’Urville, Jules, 1835. Voyage pittoresque autour du Monde, Paris, Tenré, tome II.

FIRTH Raymond with Mervyn McLean, 1990. Tikopia songs. Poetic and musical art of a Polynesian people of the Solomon Islands, Cambridge, Cambridge University Press.

JACQUES Norbert, 1922. Südsee. Ein Reisebuch, Munich, Drei Masken Verlag.

KocH Gerd, 2000. Songs of Tuvalu, Suva, Institute of Pacific Studies.

Maude Harry E., 1968. The Colonization of the Phoenix Islands, Of Islands and Men. Studies in Pacific History, Melbourne, Oxford University Press, pp. 315-342.

Moyle Richard, 2000. Collection or Theft? Germans on Takú, Pacific Arts 21-22, pp. 103-107.

PARKINSON Richard, 1889. Beiträge zur Kenntniss des Deutschen Schutzgebietes in der Südsee, Mittheilungen der Geographischen Gesellschaft in Hamburg 7 (1887-8), pp. 201-283.

—, 1907. Dreissig Jahre in der Südsee, Stuttgart, Strecker \& Schröder.

Shineberg Dorothy (ed.), 1971. The Trading Voyages of Andrew Cheyne, 1841-1844, Canberra, ANU Press.

Thode-Arora Hilke, 2001. Tapa und Tiki. Die Polynesien-Sammlung des Rautenstrauch-JoestMuseums, Ethnologica N. F. Band 23, Köln, RJM für Völkerkunde.

TreIDE Barbara, 1997. In den Weiten des Pazifik, Mikronesien. Ausgewählte Objekte aus den Sammlungen der Museen für Völkerkunde zu Leipzig und Dresden, Wiesbaden, Dr. Ludwig Reichert Verlag.

Wane Joanna, 2005. Before the Flood, Ingenio et labore, Magazine of the University of Auckland, Spring 2005, pp. 10-12. 